\title{
E-Span and LPT for data aggregation in wireless sensor networks
}

\author{
Weinan Marc Lee *, Vincent W.S. Wong \\ Department of Electrical and Computer Engineering, The University of British Columbia, 2332 Main Mall, Vancouver, BC, Canada V6T 1Z4
}

Available online 10 March 2006

\begin{abstract}
In wireless sensor networks (WSNs), when a stimulus or event is detected within a particular region, data reports from the neighboring sensor nodes (sources) are sent to the sink (destination). Data from these sources are usually aggregated along their way to the sink. The data aggregation via in-network processing reduce communication cost and improves energy efficiency. In this paper, we propose two different tree structures to facilitate data aggregation. We first propose $E$-Span, which is an energy-aware spanning tree algorithm. In E-span, the source node which has the highest residual energy is chosen as the root. Other source nodes choose their corresponding parent node among their neighbors based on the information of the residual energy and distance to the root. We also propose the Lifetime-Preserving Tree ( $L P T)$. In LPT, nodes which have higher residual energy are chosen as the aggregating parents. LPT also includes a self-healing feature by which the tree will be re-constructed again whenever a node is no longer functional or a broken link is detected. By choosing Directed Diffusion [C. Intanagonwiwat, R. Govindan, D. Estrin, Directed diffusion: a scalable and robust communication paradigm for sensor networks, in: Proc. of ACM MobiCom'00, Boston, MA, Aug. 2000, pp. 56-67.] as the underlying routing platform, simulation results show that in a WSN with 250 sensor nodes, the lifetime of sources can be extended significantly when data are aggregated by using either E-Span or LPT algorithms.
\end{abstract}

(c) 2006 Published by Elsevier B.V.

Keywords: Data aggregation; Wireless sensor networks

\section{Introduction}

The rapid advances in wireless communication and Micro Electro Mechanical System (MEMS) have made Wireless Sensor Networks (WSNs) possible. Such environments are typically comprised of a large number of sensors being randomly deployed for detecting and monitoring tasks. These sensors, developed at a low cost and in small size (mm-scale for smart dust motes [2]), are responsible for sensing, data processing, and routing activities. Applications of such networks range from battlefield communication systems (e.g., intrusion detections, target surveillance) to environmental monitoring networks such as habitat monitoring, chemical sensing, infrastructure security, inventory, and traffic control. For example, sensors may be distributed across a forest in order to report

\footnotetext{
${ }^{*}$ Corresponding author. Tel.: +1 604827 5135; fax: 16048225949.

E-mail addresses: wnmlee@ece.ubc.ca (W.M. Lee), vincentw@ ece.ubc.ca (V.W.S. Wong).
}

the origin of a fire event when there is a significant increase in the average monitoring temperature. Unlike conventional ad hoc communication networks, energy resources in WSNs are usually scarce due to the cost and size constraints of sensor nodes. Conserving energy is thus the key to the design of an efficient WSN.

Perhaps the most significant difference between an Internet-based system and a WSN is the collaborative efforts provided by sensors. Each node in an Internet-based system competes with other nodes for a fair share of resources in order to run tasks and applications of its own. Per-hop fairness is therefore the primary concern. WSNs, on the other hand, rely on the collective information provided by the sensors. Most sensor nodes are task specific in that they are all programmed for one common application. A node at one specific time may be granted more resources than other nodes if the program objective is still satisfied. For this reason, network resources are shared but it is not necessary that they be equally distributed as long as the application performance is not degraded. 
Since sensors are densely deployed in WSNs, the detection of a particular stimulus or event can trigger the response from many nearby sensor nodes. Data in WSNs are usually not directly transmitted to the interested users upon event detection. Instead, nearby sensor nodes (sources) would aggregate the data locally to remove any redundancy. Reference [3] suggests that transmitting a data packet of size $1 \mathrm{~Kb}$ to a distance $100 \mathrm{~m}$ away is similar to executing 3 million instructions on a general-purpose computer. Thus, it is beneficial to perform data aggregation via in-network processing to reduce communication cost and improve energy efficiency.

Various data aggregation algorithms have been proposed in the literature. An Energy-Aware Data Aggregation Tree (EADAT) algorithm is proposed in [4]. The base station (root) sends a broadcast control message periodically. Upon receiving this message for the first time, each node will start a timer. The expiration time is inversely proportional to the node's residual energy. The timer is refreshed when a node receives this message during the timer count down.

A dynamic convoy tree-based collaboration (DCTC) framework for tracking a mobile target is proposed in [5]. Heuristics are used to predict the object's moving direction. A dynamic tree is then created by adding or pruning the sensors near the moving target. The root of the tree can dynamically refine the readings gathered from various tree nodes.

Since the coverage area of individual sensor nodes usually overlaps, the work in [6] attempts to periodically search the smallest subset of nodes that covers the monitoring area. This group of nodes is referred to as the areadominating set. The authors in [6] proposed the use of a distributed spanning tree, induced by the initial interest flood over the area-dominating set, for aggregating reply messages from various event sources.

The problem of convergecast (many-to-one) for data aggregation is addressed in [7]. A tree that is rooted at the base station is constructed such that the link cost from each node to the base station is minimized. Further improvement includes enhancing the likelihood of simultaneous aggregation and reducing the latency for convergecast. However, the algorithm is centralized and the knowledge of global connectivity is required.

Some recent work proposed the partition of network into small adjacent grids or clusters on which data aggregation is performed. Specifically, a cluster head is associated with each cluster so that all the nodes belonging to the same cluster can aggregate their readings through this node. The work in [8] periodically selects cluster heads according to a hybrid of the residual energy and node degree, resulting in a set of energy-rich cluster heads being uniformly distributed across the network. However, their work assumes that nodes have variable transmission power to maintain a certain degree of connectivity between the clusters.

In this paper, we consider a network with $M$ randomly deployed sensors in which each node $m$ has an identical transmission range and initial residual energy $e_{m}$. An event, triggering $N$ sensors around it, occurs at a random location in the network. Data reports from these sources are clockdriven upon event detection. We define the node lifetime to be the time that a source node runs out of its energy.

We propose two different tree structures to facilitate data aggregation. We first propose E-Span, which is an energy-aware spanning tree algorithm. In E-span, the source node which has the highest residual energy is chosen as the root. Other source nodes choose their corresponding parent node among their neighbors based on the information of the residual energy and distance to the root.

We also propose the Lifetime-Preserving Tree (LPT). In LPT, nodes which have higher residual energy are chosen as the aggregating parents. LPT also includes a self-healing feature by which the tree will be re-constructed again whenever a node is no longer functional or a broken link is detected. Our proposed LPT shares the same objective as $[4,6,7]$ in an attempt to construct a data aggregation tree and select a dedicated root for which data are gathered. Both LPT and EADAT [4] select the aggregating nodes which have higher energy. In addition, LPT, EADAT [4], and HEED [8] consider the residual energy, thereby enhancing the likelihood of distributing the loads over higher-energy nodes. However, LPT differs from EADAT [4] in that extensive use of timers is not necessary. While the work in $[4,6,7]$ requires the prior knowledge or support from a given tree root, our proposed LPT does not require the root to be any particular node. In terms of root selection, we consider the residual energy of nodes within the event area whereas $[6,7]$ compare the link cost that associates with each of them.

By choosing Directed Diffusion [1] as the underlying routing platform, simulation results show that in a WSN with 250 sensor nodes, the lifetime of sources can be extended significantly when data are aggregated by using either E-Span or LPT algorithms.

The remainder of this paper is organized as follows. The network model and problem formulation are given in Section 2. In Section 3, we first present the E-Span algorithm. It is followed by the description of the centralized and distributed versions of the LPT algorithms. The performance comparisons between E-Span, LPT, and Directed Diffusion are presented in Section 4. Conclusions are given in Section 5.

\section{Background}

In this section, we first present the network model under considerations, the related definitions and assumptions. We then describe the problem formation and provide an overview of the spanning tree algorithm.

\subsection{Network model}

We consider a field of $M$ randomly deployed and identical sensor nodes. A number of $K(K<M)$ sinks, randomly 
chosen among these $M$ nodes, are requesting for data reports. A stimulus, triggering $N(N<M)$ event sources around it, occurs at a random location in this field. We assume that these sources are interconnected to each other. In practice, an event may not trigger a set of connected source nodes. However, under such scenario, multiple independent trees will be constructed with each serving a disjoint set of event sources. Hence, for simplicity, we restrict ourselves to a set of $N$ connected source nodes. We assume that data reports from each source are clockdriven upon event detection. Furthermore, these data are assumed to be collected at a dedicated tree root and sent to the distant sinks in a periodic manner. During data collection, nodes have the abilities to perform in-network aggregation of packets enroute to the tree root. We further assume that each node $m(m \in\{1,2, \ldots, M\})$ is aware of its energy, $e_{m}$. Node batteries are neither replaceable nor rechargeable. We finally assume that all nodes have an identical and fixed transmission range. We define a branch to be the route from a root node to a leaf node in a given tree. The following two terms are introduced:

Branch energy - the minimum energy of all the non-leaf nodes in a given tree branch.

Tree energy - the minimum branch energy of all the branches in a given tree.

Let $B_{y}$ denote the set of nodes along a given tree branch with $y$ as the leaf node, and $I_{x}$ be the set of nodes in a given tree rooted at node $x$. Mathematically, the branch and tree energies are calculated as follows:

Branch energy for branch $B y=\min _{i \in B_{y}, i \neq y}\left\{e_{i}\right\}$,

Tree energy for tree $I_{x}=\min _{j \in I_{x}, j \neq \text { leafnode }}\left\{e_{j}\right\}$.

An example of the branch energy and tree energy calculation is given in Fig. 1.

\subsection{Problem formulation}

Given a number of $N$ connected source nodes with each source labeled $n(n \in\{1,2, \ldots, N\})$ and the knowledge of their own residual energy, $e_{n}$, our goal is to find a tree spanning all these sources and an appropriate tree root for data collection so that the functional lifetime of each source is preserved as much as possible. Note that the time till the first link breaks in a given tree determines the lifetime of each source (due to the additional energy cost for the broken-link repairs), and the tree energy directly reflects this time. We hence tackle this problem by searching for a tree with the highest tree energy.

In the literature, network lifetime has often been defined as either the time till the first or a set of nodes runs out of its energy [9-12], or till the first loss of connectivity or coverage [13,14], or a combination of these [15]. A formal definition of network lifetime may depend on the application
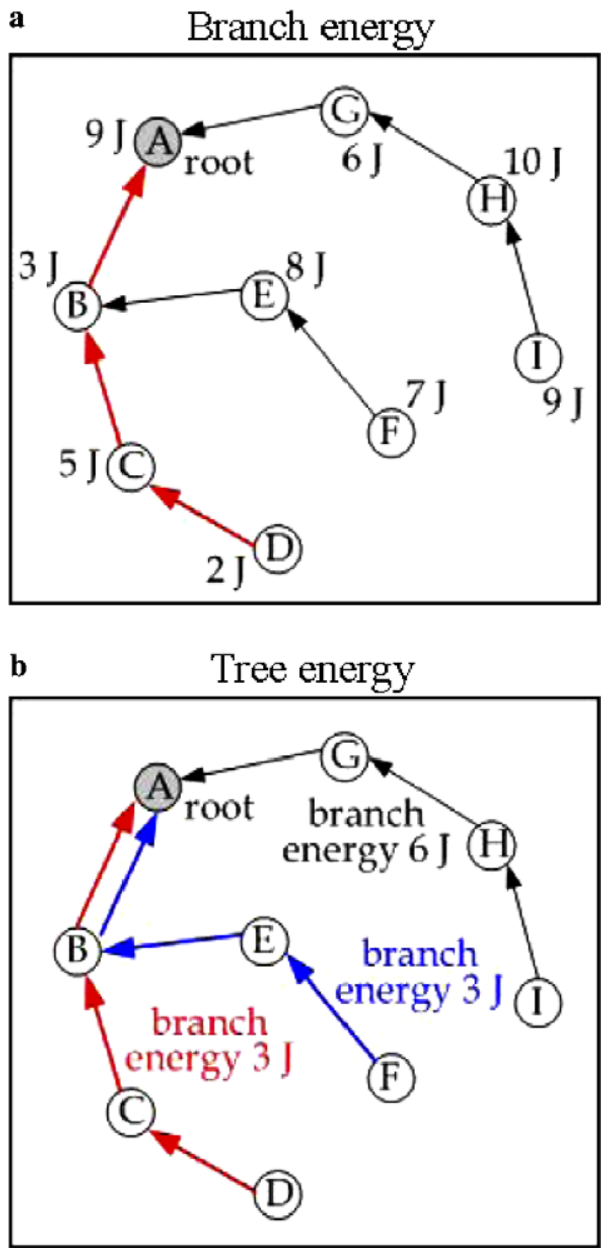

Fig. 1. An example to describe branch and tree energies: (a) energy of the branch from nodes $\mathrm{A}$ to $\mathrm{D}$ is set to $3 \mathrm{~J}$, the energy of node $\mathrm{B}$. (b) Tree energy is set to $3 \mathrm{~J}$, the energy of the branch from either nodes A to D or nodes $\mathrm{A}-\mathrm{F}$.

scenario in which the network is targeted at. However, none of these definitions deviate from interpreting network lifetime as the time before the network ceases to provide the type of service it is designed for. We therefore follow this convention, and define the branch energy as the minimum energy of all the non-leaf nodes in a given branch and define tree energy as the minimum branch energy of all the branches in a given tree. Note that the time that an upstream link along a given branch takes to break depends on the energy of the parent on such a link. In other words, the time during which data from each source along a given branch can arrive at the root depends on the minimum energy of any parent along this branch. The only remaining question is to determine how to select an appropriate tree root and the branch leading to each other source, such that the energy of this tree is maximized.

In this paper, we propose two different tree structures to facilitate data aggregation. The first algorithm, called E-Span, is a variation of the spanning tree algorithm. An overview of the original spanning tree algorithm is given in the next section. 


\subsection{Spanning tree algorithm}

A spanning tree is a graph that spans all the nodes as vertices and contains no cycles. The tree is structured in a way that the node with the smallest identifier is chosen as the root. At the same time, all other nodes are connected to this selected root via the shortest-path route. The protocol requires each node to exchange configuration messages in a format that contains the identifier of itself, that of its selected root, and the distance (in hops) to this selected root. Each node updates its configuration message upon identifying a root with a smaller identifier or the shortestpath neighbor. Furthermore, the neighbor for which the shortest-path configuration message comes from is chosen as the parent of a node whenever it is detected. A node identifier is used to break ties if necessary.

The above descriptions are being translated into the GetSpan algorithm depicted in Table 1. Note that the term single-hop broadcast refers to the operation of sending a packet to all single-hop neighbors. Lines 1 and 2 restrict the message exchanges to within the event region. Line 3 starts the exchange and an additional timer for tree maintenance. Line 4 triggers an infinite loop. Lines 5-9 allow a root to periodically generate a message every $T$ seconds and reset a node when it starts to lose its shortest-path neighbor. Lines $10-15$, on the other hand, update the node itself and forward the message whenever a node identifies a root with a smaller identifier or a better shortest-path neighbor.

For example, the set of sources depicted in Fig. 2(a) will create a tree of the form shown in Fig. 2(b). Unfortunately,

Table 1

The distributed spanning tree protocol

\begin{tabular}{|c|c|}
\hline Define: & $\begin{array}{l}r_{n} \text { to be the ID of the root selected by node } n \\
d_{n} \text { to be the shortest-path distance from } r_{n} \text { to node } n \\
g_{n}=\left(n, r_{n}, d_{n}\right) \text { to be the message sent by node } n \\
p_{n} \text { to be the ID of the parent selected by node } n \\
t_{\text {rec } n, n} \text { to be the time node } n \text { received the message } \\
\text { from its parent }\end{array}$ \\
\hline Initialize: & $\begin{array}{l}g_{n} \text { to }(n, n, 0) \forall n \in N \\
p_{n} \text { to } n \forall n \in N \\
t_{\text {recv }, n} \text { to } 0 \forall n \in N\end{array}$ \\
\hline \multicolumn{2}{|c|}{ GetSpan (node ID $n$, time $t$, timeframe $T$ ) } \\
\hline 1 & if $(n !=$ event source $)$ \\
\hline 2 & return; \\
\hline 3 & $\begin{array}{l}\text { single-hop broadcast } g_{n} \text { and start a timer } P \text { that expires } \\
\text { every } T \text { sec; }\end{array}$ \\
\hline 4 & while true \\
\hline 5 & if $P$ expires and $\left(r_{n}=n\right.$ or $\left.t>t_{\text {recv }, n}+T\right)$ \\
\hline 6 & set $g_{n}$ to $(n, n, 0)$ \\
\hline 7 & set $p_{n}$ to $n$ \\
\hline 8 & set $t_{r e c v, n}$ to $t$ \\
\hline 9 & single-hop broadcast $g_{n}$ \\
\hline 10 & if receiving a message $g_{i}$ from node $i$ \\
\hline 11 & $\begin{array}{l}\text { if } r_{i}<r_{n} \text { or }\left(r_{i}=r_{n} \text { and } d_{i}+1<d_{n}\right) \text { or }\left(r_{i}=r_{n} \text { and }\right. \\
\left.d_{i}+1=d_{n} \text { and } i \leqslant p_{n}\right)\end{array}$ \\
\hline 12 & set $g_{n}$ to $\left(n, r_{i}, d_{i}+1\right)$ \\
\hline 13 & set $p_{n}$ to $i$ \\
\hline 14 & set $t_{r e c v, n}$ to $t$ \\
\hline 15 & single-hop broadcast $g_{n}$ and restart timer $P$ \\
\hline
\end{tabular}

a Connectivity diagram

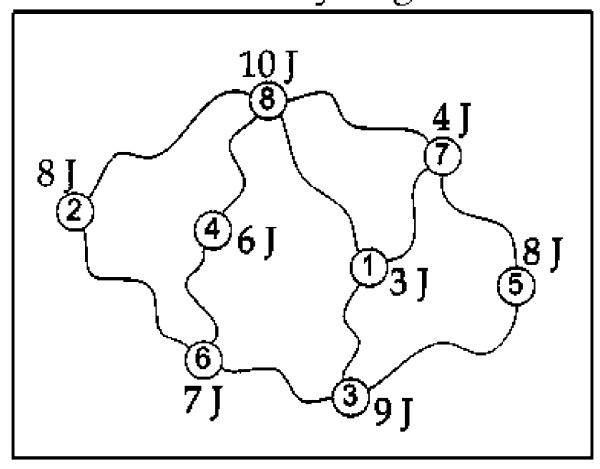

b Spanning tree configuration

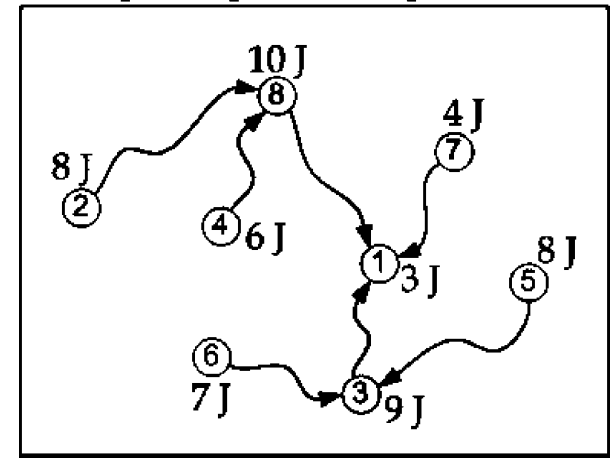

Fig. 2. An example of the spanning tree protocol: (a) connectivity diagram for a set of given sources. (b) The spanning tree configurations will have node 1 with energy $3 \mathrm{~J}$ chosen as the root, resulting in the lowest tree energy of $3 \mathrm{~J}$.

failure to consider the node's residual energy results in this tree having the lowest energy of $3 \mathrm{~J}$. Furthermore, the node that is equipped with the minimum energy, i.e., node 1 , is chosen as the root and is attached to three other child nodes. When this tree is deployed for data collection among these sources, the rate at which node 1 dissipates its energy is quite high and thus the time to the first node death is minimized. We therefore make some changes and present an energy-aware variant of this protocol, namely E-Span.

\section{Proposed tree structures}

In this section, we describe two different tree structures to facilitate data aggregation. We first describe the E-Span algorithm $[19,20]$. It is followed by the description of the centralized and distributed versions of the LPT algorithm [21]. Unless stated otherwise, we will use the connectivity diagram shown in Fig. 2(a) for all the examples illustrated in this section.

\subsection{E-Span}

As with the conventional spanning tree, E-Span is a graph that covers all the nodes as vertices and contains no cycles. All other nodes are still connected to the selected 
root via the shortest-path route. Since the root, besides collecting data, is also responsible for coordinating the routes with distant sinks, the node with the highest energy level is now chosen as the root. Moreover, any other node is given the choice to select its parent as the highest-energy neighbor for which the shortest-path message comes from. By using the connectivity diagram in Fig. 2(a), the tree will now have node 8 chosen as the root and all other nodes are connecting to node 8 via the shortest-path route (see Fig. 3). Specifically, node 6 which finds itself having two shortest-path neighbors of nodes 2 and 4 will in fact attach itself to the higher-energy one (i.e., node 2). The reason is to allow a node that has more available resources to be selected as a parent node for data collection.

The pseudo-code of the E-Span algorithm is given in Table 2. The configuration message now involves three additional parameters: the residual energy of the node that sends the message, the residual energy of the node's chosen root, and the residual energy of the node's chosen parent. As with the GetSpan algorithm, lines 1-3 start the message exchanges and restrict these exchanges to within the event area. Lines 4-7 allow a root to periodically generate a message every $T$ seconds and reset a node that loses connection with its parent. Lines 8-12 update the list of child nodes for the receiving node. Lines 13-17 update the message when a node receives an energy update from its parent, or when it detects a better shortest-path neighbor or a higher-energy root. Lines 18 and 19 compare the receiving node with the root. Lines 20 and 21 broadcast the message after a change. Again, single-hop broadcast is referred to the operation of sending a packet to all single-hop neighbors.

Unfortunately, without knowing the complete set of connectivity provided by all sources, some nodes in E-Span still traverse to the root through routes with a lower branch energy. As a result, each source can more often be involved in tree re-construction, implying that a greater portion of its available energy is consumed in repairing broken tree links over the course of its lifetime. As an example (see Fig. 3), nodes 3 and 5 could have been attached to nodes 6 and 3, respectively, resulting in tree energy of 7 rather than $3 \mathrm{~J}$. In addition, the energy dissipation rates for nodes

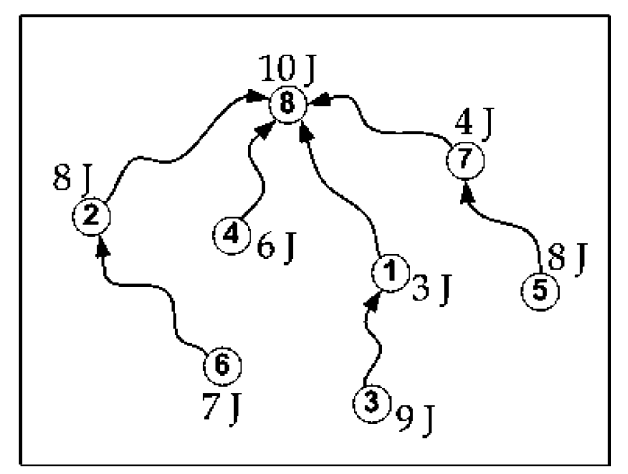

Fig. 3. An example of the E-Span protocol. The E-Span configurations will have node 8 with energy $10 \mathrm{~J}$ chosen as the tree root, still resulting in the lowest tree energy of $3 \mathrm{~J}$.
Table 2

The distributed energy-aware spanning tree protocol

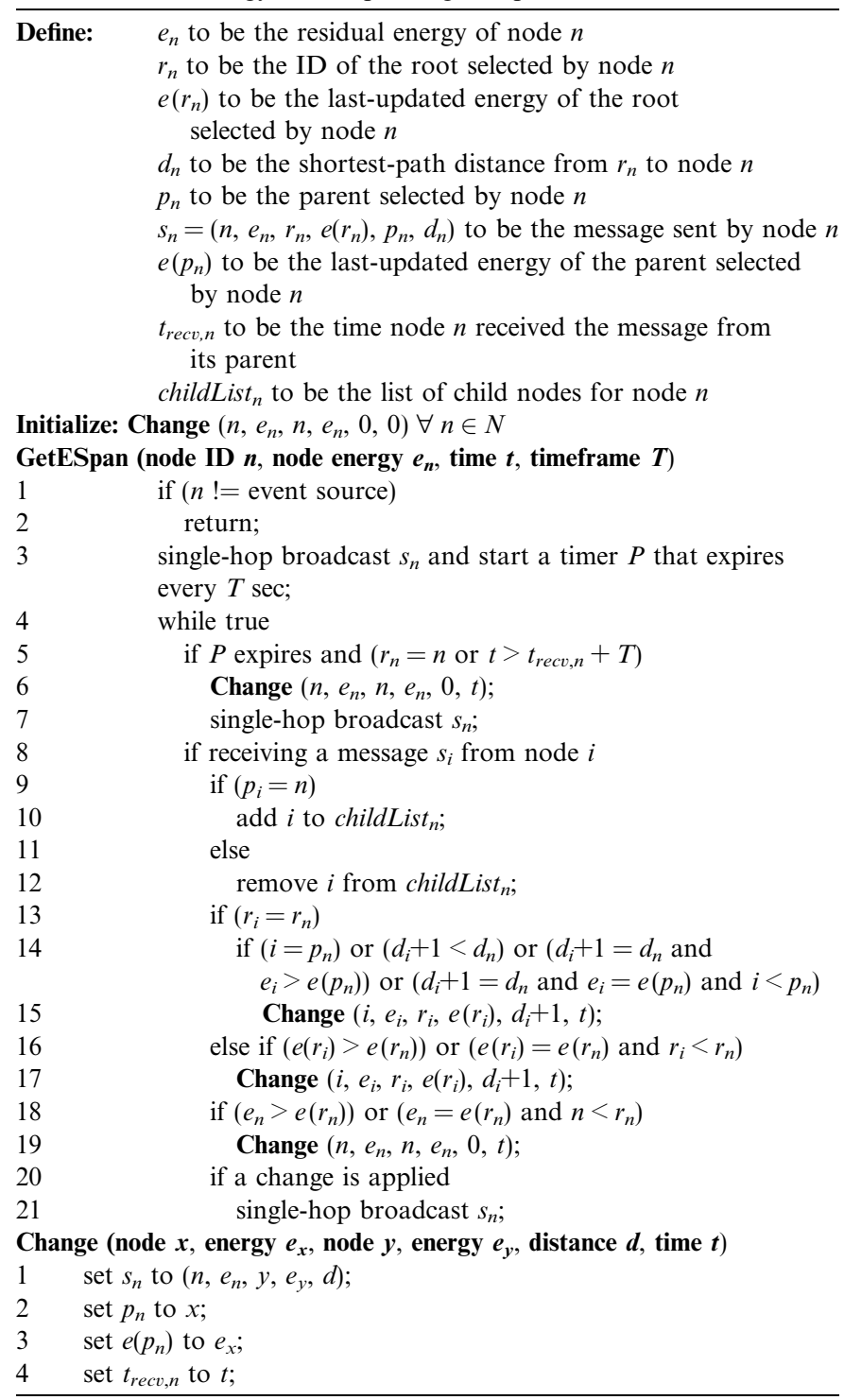

1 and 7 would have been lower if the above changes were made. In other words, without making these changes, the functional lifetime of the two nodes are shorter due to the additional energy cost involved in the tree re-construction. This is clearly one issue we try to resolve, if possible, when we construct the LPT. However, we believe that the chance for it to happen is rather rare for a small number of participating sources. When the number of source nodes is large, a technique that is different from E-Span is required. We shall now present our LPT construction algorithm.

\subsection{LPT: the centralized approach}

In this section, we proceed to the discussion of the lifetime-preserving tree (LPT) construction using a centralized approach. We assume that complete knowledge of the event area is given, including the connectivity and residual 
energy of all the source nodes, prior to the start of the algorithm. The tree generated can later be used to validate the correctness of the corresponding tree constructed by the distributed approach.

One way to obtain an LPT is to directly run an extensive search at every node and then compare various tree energies. However, this approach has the scalability problem when the number of source nodes is large. We hence tackle the issue in a different manner.

Recall that the LPT requires a root (initially unknown) to collect data from each other node via routes with the highest branch energy, subject to the condition that these routes do not create loops. Moreover, the tree has an energy that directly depends on the minimum residual energy of all non-leaf nodes. If there exists a way to identify this minimum-energy node, which represents the bottleneck to the network, it will then be easy to determine what the highest tree energy will be.

To illustrate the above description, consider the set of nodes in Fig. 2(a). Any source node can either be a root, parent, or leaf. By assuming that node 5 is a root, the protocol must have each other node enroute to this root through either node 7 or 3 . However, the tree must have node 6 as a parent for some nodes in the network if the tree energy has to be the highest (depicted in Fig. 4(a)). If node 5 is now a parent, node 6 again has to forward data for some nodes, for example nodes 2 and 4, in the network (Fig. 4(b)). Finally, if node 5 is a leaf, the protocol must use node 3 to forward its data to some root via either node 1 or 6 . By using the same argument, node 6 again has to be a parent for data collection from node 3 (shown in Fig. 4(c)). We therefore consider node 6 to be the bottleneck node for this particular network topology, and the tree must have energy less than that of this node.

We now address the question of how to identify this bottleneck node and coordinate the given set of network connection such that a tree is obtained with this node being configured as the minimum-energy non-leaf node. We begin by arranging nodes in ascending energy levels. Starting from the least-energy node, we test if the removal of all network links to this node except that from its highest-energy neighbor will disconnect the existing graph. If so, the bottleneck node is found and there are no better ways than to collect data via this node. The removed links are thus restored, and any tree rooted at one of the nodes in the remaining set shall have the energy as that of this chosen node. If not, the removed links do not contribute to the construction of the lifetime-preserving tree and we shall move on to the next node. Note that the energy of the highest-energy neighbor has to be greater than that of the node under the test. When such neighbor does not exist, the node has to be a parent for at least one of its neighbor, and thus all the links are preserved. In the case when there are more than one neighbors that have equal highest energy, either one can serve as the parent for collecting data from the node under the test without affecting the tree energy. Node ID is thus used to break this tie. Finally, for the last node,

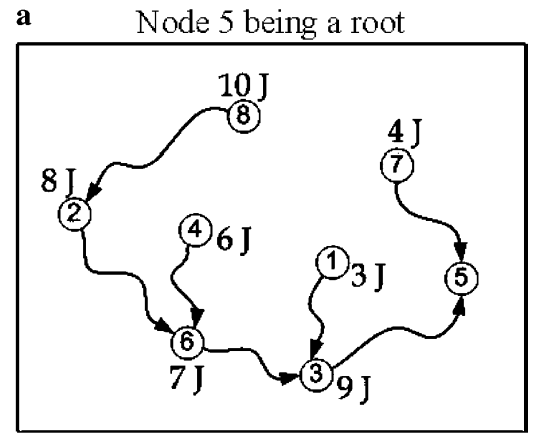

b Node 5 being a parent
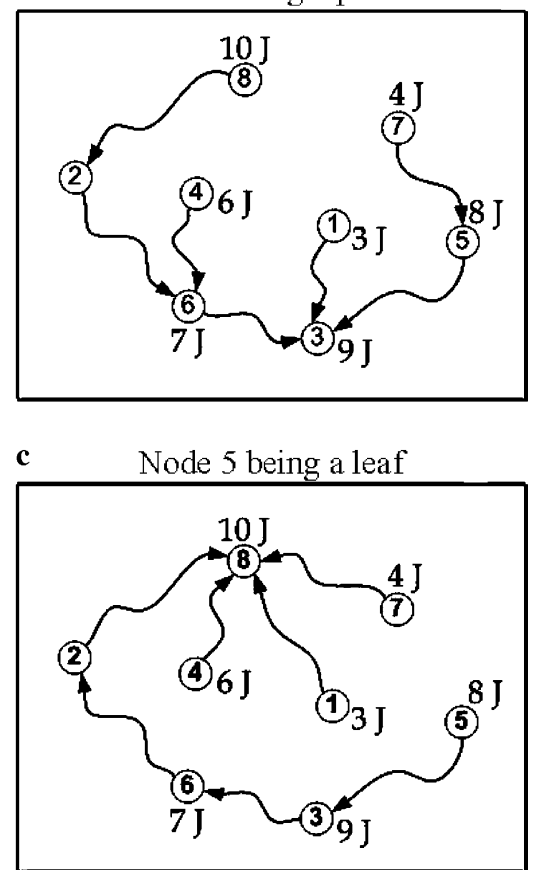

Fig. 4. An example of bottleneck node. (a) If node 5 is a root, node 6 has to collect data from nodes 2 and 4. (b) If node 5 is a parent, node 6 again has to forward data for both nodes 2 and 4. (c) If node 5 is now a leaf, node 6 has to collect data from node 3 . Therefore, the tree energy cannot be greater than the energy of this bottleneck node.

i.e., the highest-energy one, we conclude that there is no bottleneck node for this particular topology and any tree rooted at this last node, on the existing graph, can have the highest tree energy.

To illustrate the descriptions, consider two examples. Fig. 5 depicts the centralized LPT search during which a bottleneck is found when the link from nodes 6 to 2 is removed. In other words, node 6 has to be a parent for some nodes in the network. Any tree rooted at one of the nodes in the remaining set, i.e., nodes $2,3,5,6$, or 8 , will therefore have the highest tree energy as that of this bottleneck node. Fig. 6 depicts another example of the centralized search where no bottleneck node is found. The reason is that those links that are removed do not contribute to the formation of any lifetime-preserving tree and therefore any tree rooted at the highest-energy node, i.e., node 2, will have the highest tree energy. 
a

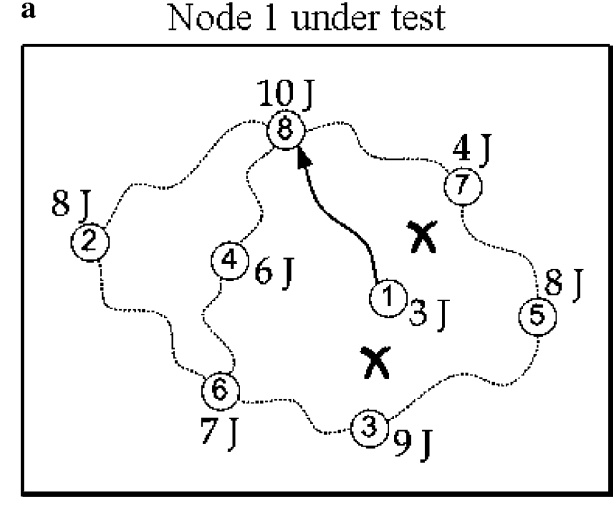

b
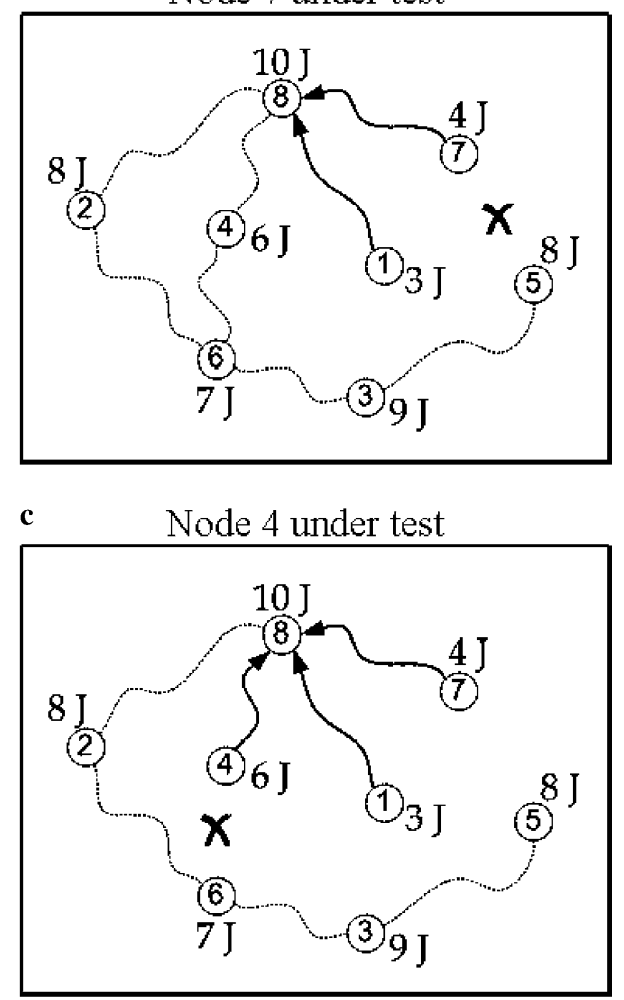

d

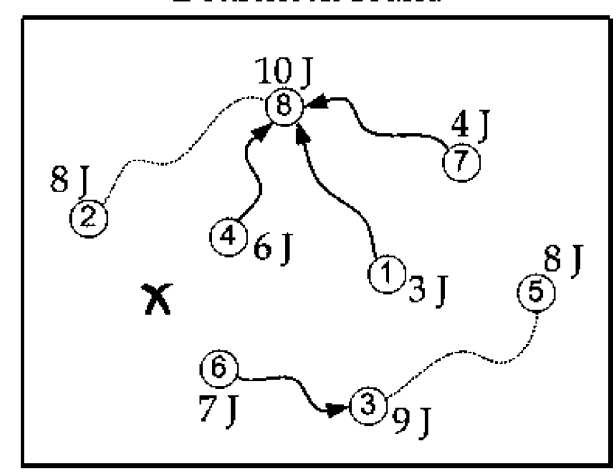

Fig. 5. An example of the centralized LPT search. Starting from the leastenergy node, we test if the removal of all the links to this node except that from its highest-energy neighbor will disconnect the existing graph. For this topology, node 6 is hence found as the bottleneck. Any tree rooted at nodes $2,3,5,6$, or 8 , on the existing graph will have the highest tree energy of $7 \mathrm{~J}$.
The pseudo-code of the centralized version of the LPT algorithm is given in Table 3. Line 1 sorts all nodes in ascending energy levels. Lines 2 and 3 compute the highest-energy neighbor for the node under the test. Recall that the energy of this neighbor has to be greater than that of the node. When such a neighbor exists, lines 4 and 5 remove and temporarily store all links to the node except that from the highest-energy neighbor. Lines 6-10 restore the removed links, clear the storage, and compute a tree by running Dijkstra's algorithm [16] at one of the nodes in the remaining set when a bottleneck is found. Tree energy is set to the energy of the bottleneck node at this time. In fact, the reason to run the Dijkstra's algorithm is to ensure that the remaining set of network connections does create a tree and contain no loops. Lines 11-12 compute a tree again by running Dijkstra's algorithm at the highest-energy node, and search the tree energy by using Eq. (2) when no bottleneck node exists in the network.

\subsection{LPT: the distributed approach}

Given a number of $N$ source nodes with each node labeled $n(n \in\{1,2, \ldots, N\})$ and the knowledge of their own residual energy, $e_{n}$, our goal is to construct a tree spanning all these sources and to select an appropriate root for data collection, in a distributed way, such that the energy of the tree is maximized. We take the approach of exploring the highest-energy branch from each source to a root, by first assuming that every source node is a root, using a method similar to Reverse-Path Forwarding (RPF) [17]. This generates a total of $N$ unique trees with each being rooted at a distinct source node. We continue by comparing the energy of these trees and only employ the one with the highest tree energy for data collection.

\subsubsection{Exploring the highest-energy branch from every source to any root}

As previously mentioned, the time during which data from each source along a given branch can actively be received by the root depends on the minimum energy of any parent along this branch. In order to maximize this time for any pair of root and source, the connectivity between them will first have to be explored prior to obtaining the highest-energy branch connecting these two nodes. Let $P_{x, y}$ denote the set of possible routes, with each labeled $p$, from nodes $x$ to $y$ and $b r E_{i, j, k}$ be the energy of a branch $k$ with node $i$ as the leaf and node $j$ as the root. Note that $k \in P_{i, j}$. We determine a branch $b$ for every pair of source $s$ and root $r$ such that

$b r E_{s, r, b} \geqslant b r E_{s, r, p} \forall p \in P_{s, r}, p \neq b$.

We use RPF which requires each source $s$ to initiate a configuration message in a format that contains its energy information. When a source receives this message, it appends its energy information and broadcasts the message only if it has not seen this message or if it has previously forwarded the message containing lower branch energy. 
a

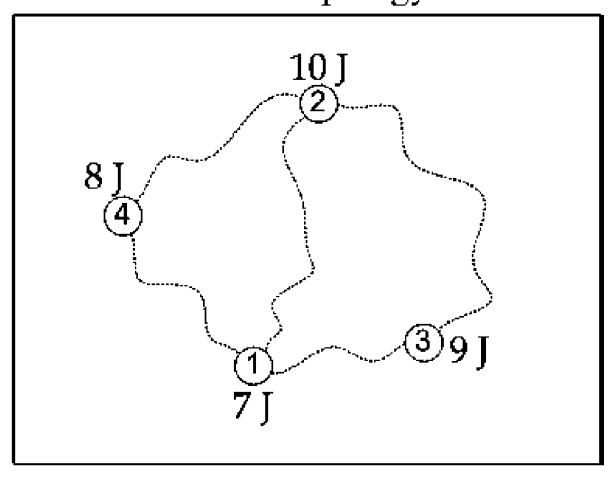

b

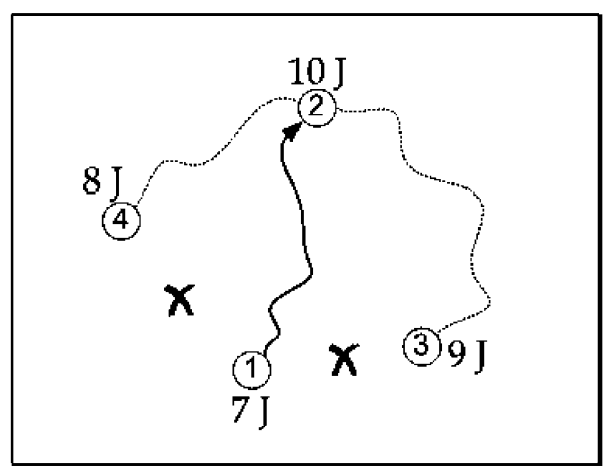

c

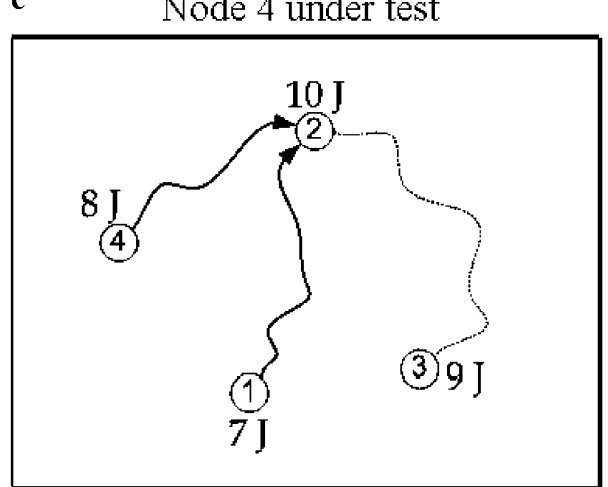

d

Bottleneck not found

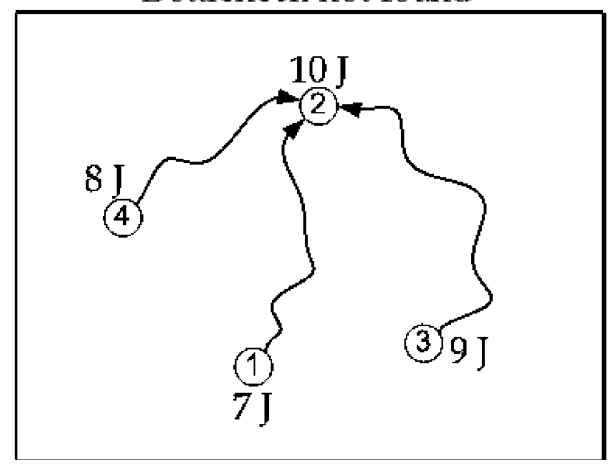

Fig. 6. An example of the centralized LPT search. Since removal of the links, except that from the highest-energy neighbor, to the source under any test does not disconnect the existing graph, there is no bottleneck for this particular network topology. Hence, any tree rooted at node 2 will be the lifetime-preserving tree.
Table 3

The centralized LPT algorithm

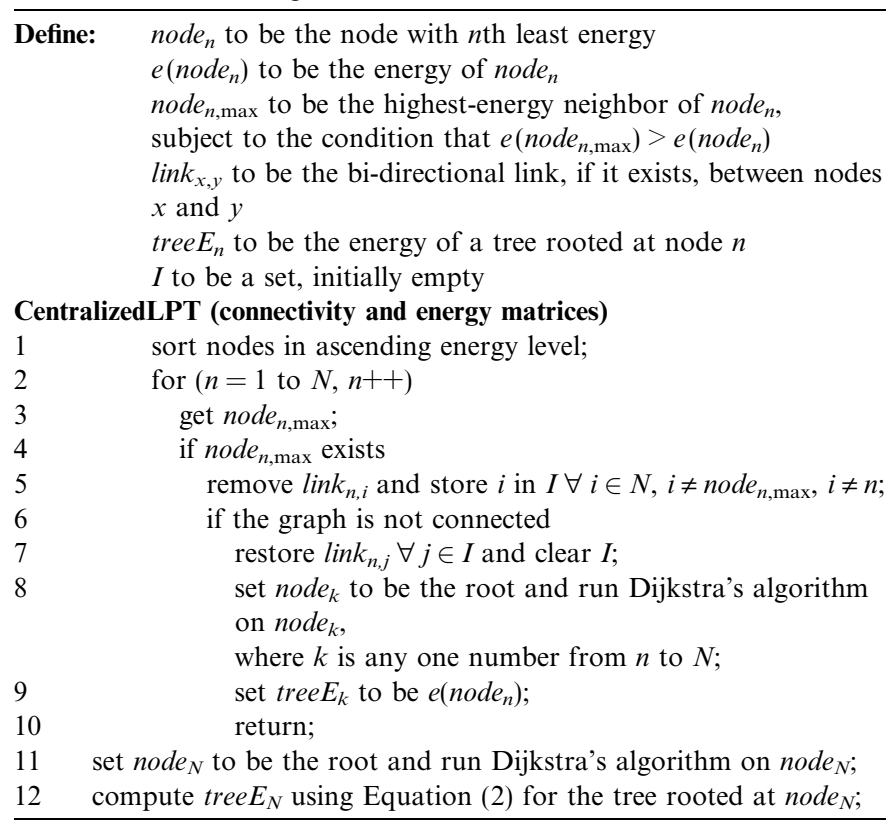

Otherwise, it simply discards the received packet. Eventually, various copies of the initiated message will traverse through various different routes $p$ and only the better ones will arrive at the root $r$.

We define $e i d_{n}$ to be the pair of energy level and ID of a node labeled $n$ and brList $_{i, j, k t o}$ be a list containing the eid for the message initiating node $i$ up to the last receiving node $j$ via a route $k$ with branch energy $b r E_{i, j, k}$. Note that $b r E_{i, j, k}$ can be calculated by using Eq. (1). Therefore, brLis$t_{i, j, k}$ shall have the format of a list as follows:

brList $_{i, j, k}:$ eid $_{i} \rightarrow \operatorname{eid}_{x} \rightarrow \ldots \ldots \rightarrow$ eid $_{y} \rightarrow e i d_{j}$,

where nodes $x$ and $y$ are the intermediate receiving nodes for the message initiated by node $j$. Note that when node $j$ receives the list $b r$ List $_{i, y, p}$ from node $y$ via some route $p$, it is as if node $j$ is a root and node $i$ is a leaf for the branch between nodes $j$ and $i$. Our descriptions can thus be translated into the ExploreBranch function shown in Table 4. Again, single-hop broadcast refers to the operation of sending a packet to all single-hop neighbors. Line 1 allows each source to initiate its control message. Lines 2-5 update, store, and broadcast the message when the receiving node does not recognize the initiating source. Lines 6-9 reset its stored list in addition to the above three actions whenever the receiving node detects a higher-energy branch.

To illustrate the ExploreBranch function, consider the set of sources shown in Fig. 7. For simplicity, we will concentrate on the control message exchanges between a source of node 5 and a root of node 8 . First, the function requires node 5 to initiate a control message containing its eid. There are six possible routes the message could have possibly traveled (depicted in Figs. 7(a)-(f)). Among these routes, only nodes 7 and 1 will drop the messages with the routes through nodes 1 and 3, and node 7, respectively, 
Table 4

The ExploreBranch function

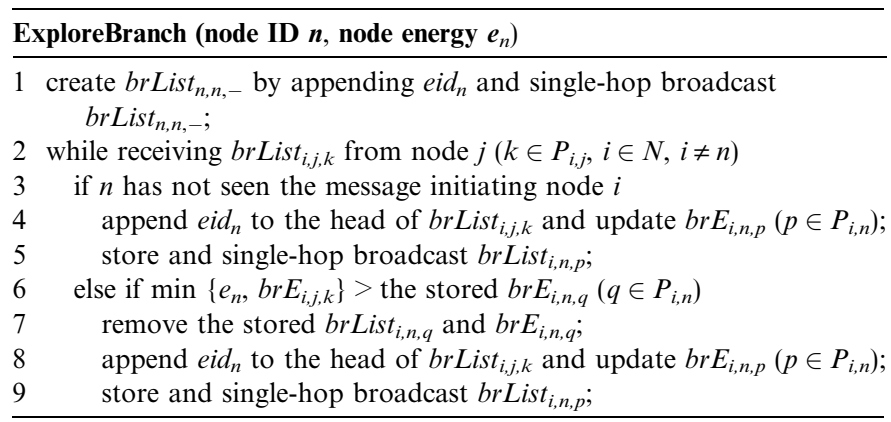

a

a $\quad$ Route through node 7

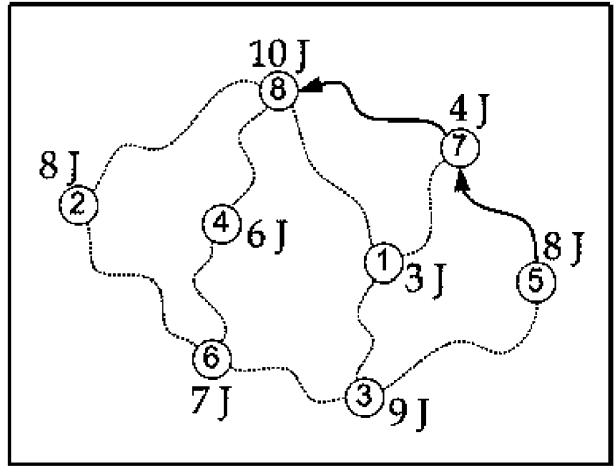

c Route through node 4,6 , and 3

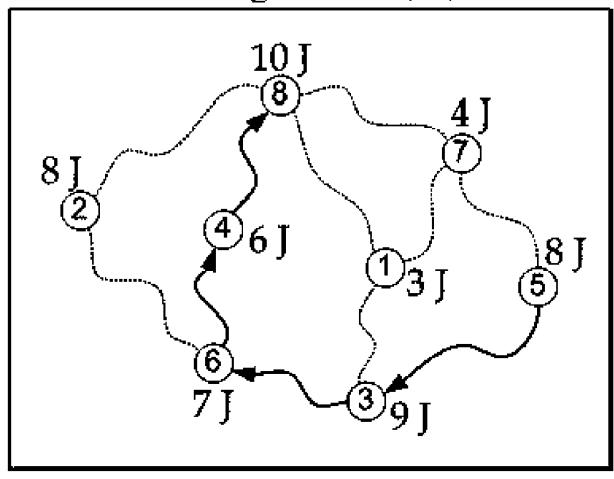

e $\quad$ Route dropped at node 7

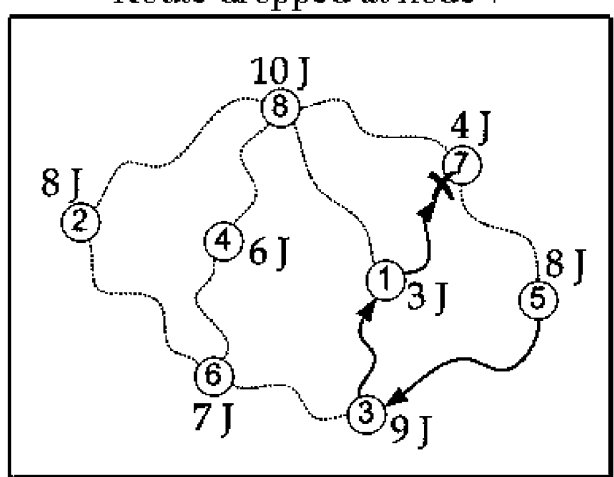

subject to the condition that they have previously forwarded the messages, containing lower branch energy, initiated by node 5 . The reason to drop these messages is to limit the number of control message exchanges and ensure that only the better routes will traverse through the nodes. Node 8 , upon receiving the other 4 routes, will be able to identify that the route from node 5 through nodes 2, 6, and 3 indeed has the highest branch energy of $7 \mathrm{~J}$.

\subsubsection{Constructing a tree spanning all event nodes for every source}

We now proceed to construct $N$ trees with each tree rooted at a distant source by incrementally attaching any

b Route through node 1 and 3

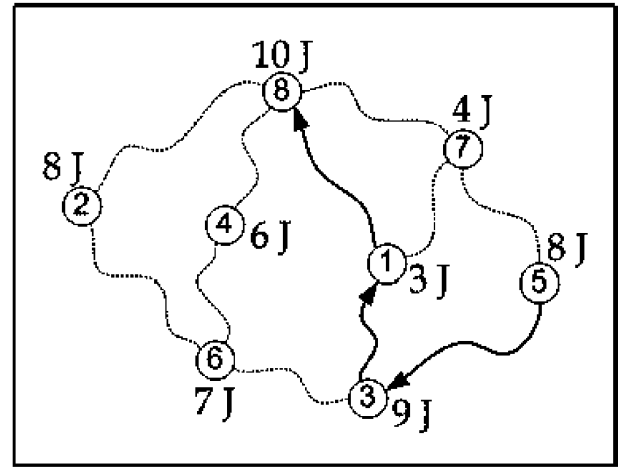

d Route through node 2, 6 , and 3

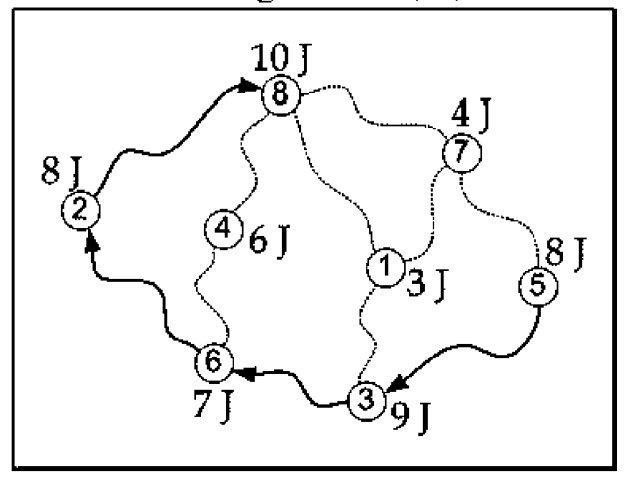

f Route dropped at node 1

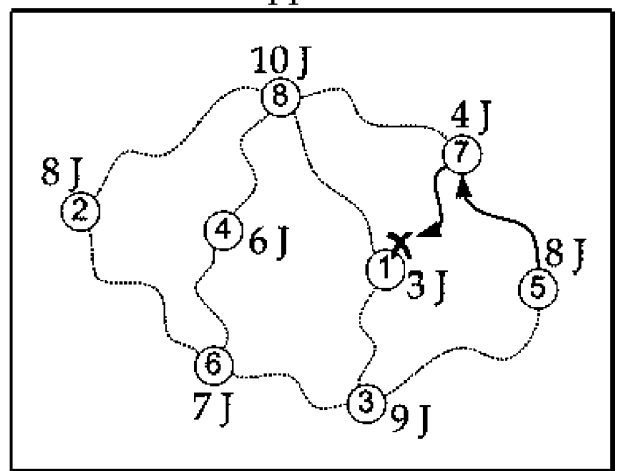

Fig. 7. An example of the ExploreBranch function. There are 6 possible choices in which only the route in (d) will have the highest branch energy of $7 \mathrm{~J}$. The messages traveling on the last two routes are dropped since they do not carry higher branch energy, subject to the condition that nodes 1 and 7 have previously forwarded a similar message. 
branch explored in the last section. Each source has an initial tree structure that only comprises the node itself. In order to construct a tree for each source that spans all event nodes, each source has to incrementally update its existing tree structure upon receiving any branch with an unknown initiating node. Note that the energy of the received branch directly determines the energy of the updated tree. To ensure that each tree carries the highest energy, the tree is also updated whenever the receiving node identifies a message with higher branch energy.

We define the term, initiator, to be the source which initiates the message, and tree $_{n}$ to be the tree created by node $n$. In other words, tree $_{n}$ stores the incoming branches received by node $n$. Also let $J_{j}$ denote the set of initiators in tree $_{j}$ and tree $_{k}$ be the energy of tree $_{k}$. tree $_{k}$ can be calculated by using the following equation:

tree $E_{k}=\min _{j \in J_{k}}\left\{b r E_{j, k, p}\right\}, \quad$ where $p \in P_{j, k}$.

Our description can be translated into the NoLoop function shown in Table 5. This function takes the received branch as an input and tests whether attaching it to the tree will not create a loop. Line 1 removes the message initiator $i$ so that the branch only contains a list of parents. Line 2 ensures the already-attached branch for node $k$ stored in tree $_{n}$ matches the route through which the received branch travels. Note that the function always accepts a branch of a size less than 3 since a loop can only be created by adding a branch of a size greater than 2 to its existing tree.

\subsubsection{Constructing a tree spanning all event nodes for every source}

Since each source $n$ carries its unique tree structure stored in $t_{r e e}$, the protocol requires every source to broadcast its tree and select the one with the highest tree energy for data aggregation among these nodes. Our objective is to create a tree rooted at node $r$ such that:

treeE $_{r} \geqslant$ treeE $_{n} \forall n \in N, n \neq r$

Note that tree $_{r}$ and tree $E_{n}$ can both be calculated using Eq. (5). In fact, there can be multiple different trees that yield the same tree energy. To break such ties, a number of other properties can be compared (e.g., tree depth, root energy, root ID, and node degree). Tree depth can be used to minimize the data latency. Root energy can be used to maximize the available resource of the root for possible tasks such as route coordination to distant sinks. Node degree can be used to minimize the power dissipation rate.

Table 5

The NoLoop function

\begin{tabular}{|c|c|}
\hline \multicolumn{2}{|c|}{ NoLoop (node ID $n$, branch brList $_{i, j, k}$ ) } \\
\hline 1 & 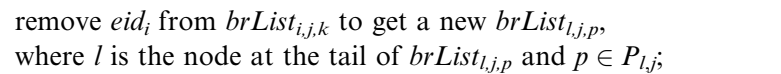 \\
\hline 2 & $\begin{array}{l}\left.\text { if number of eids in } \text { brList }_{l, j, p}<2 \text { or } \text { (brList }_{l, j, p} \| \text { eid }_{n}\right)=\text { brList }_{l, n, p} \\
\text { (stored at } \text { tree }_{n} \text { ) }\end{array}$ \\
\hline 3 & return true; \\
\hline 4 & return false; \\
\hline
\end{tabular}

In this work, we limit ourselves to use tree depth, root energy, and then root ID to break ties whenever necessary. Further work is required to evaluate the performance of the best tree selection by using other parameters.

To illustrate the descriptions, consider Fig. 8. By having each participating node broadcast its selection, there will be 8 trees under the comparison. Among them, only the ones constructed by nodes $2,3,5,6$, and 8 comprise the highest tree energy of $7 \mathrm{~J}$. We therefore wish to select the tree created by node 3 as our lifetime-preserving tree and node 3 as our root since this tree has a lower tree depth than that by nodes 5 and 8 , and a higher root energy than that by nodes 2 and 6 .

We define $l p t_{i}$ to be the lifetime-preserving tree that a node $i$ currently selects and $l p t E_{i}$ be the energy of $l p t_{i}$. Note that $l p t_{n}$ initially equals to tree $_{n}$ for all sources $n$. The Search $L P T$ function shown in Table 6 describes the procedures to search a lifetime-preserving tree for each source. Line 1 allows the source to broadcast its initial selection. While a message is received from its neighboring node with tree information (line 2), lines 3-5 update the selection of the receiving node if the received tree is better. Line 6 rebroadcasts the selection after the update. Note that we have explicitly assumed that each node has already created a tree spanning all event sources (i.e., each tree has $N$ rows) before the function starts. Hence, the first two lines of the BetterTree function are always skipped. In practice, the number of event sources is not known a priori and each node will simply run a new Search $L P T$ function whenever it detects a new initiator. To ensure that only the selections with $N$ number of rows in them are compared, we include the first two lines of the BetterTree function in order to filter out all outdated trees.

\section{Performance evaluation}

In this section, we present the performance comparisons between E-Span [20], LPT [21], and the Directed Diffusion [1].

\subsection{Simulation model}

We implemented our tree construction modules on top of Directed Diffusion [1] in the $n s-2$ network simulator [18]. A square field with each side measuring $L$ meters is considered. A number of $M$ identical nodes, ranging from 50 to 250 in increment of 50, are randomly deployed in this field such that the average node density is kept at $\lambda=50$ / $160^{2}$ nodes $/ \mathrm{m}^{2}$. Also, 5 sinks and $N$ sources are randomly chosen among the nodes, subject to the conditions that $N=0.1 \mathrm{M}$ and sources be interconnected to each other (to model a single stimulus). The radio range is $40 \mathrm{~m}$. To model an event-driven data network, each source generates random data reports of a size fixed at 136 bytes in constant intervals 1 of packet/s. An application that computes the average of reports generated by various sources is used to model the aggregation behavior. 
a Tree constructed by node 1

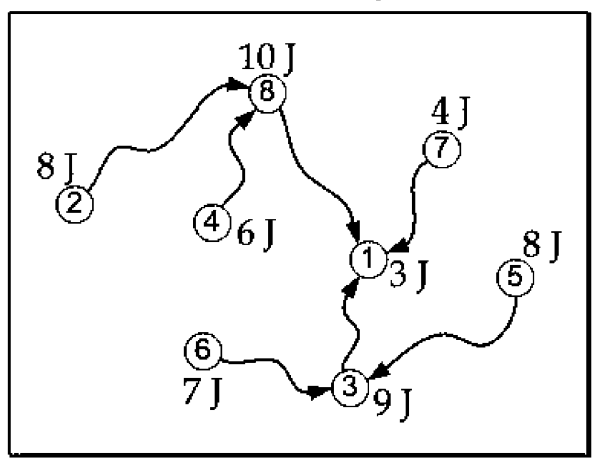

c Tree constructed by node 3

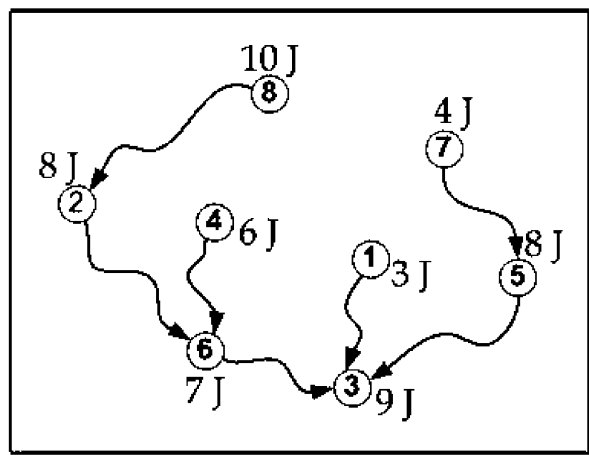

e Tree constructed by node 5

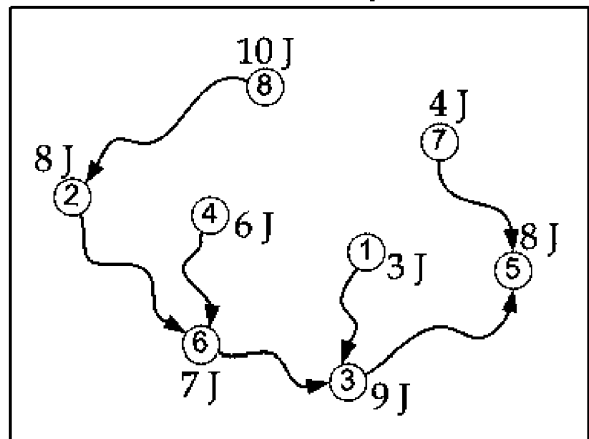

g Tree constructed by node 7

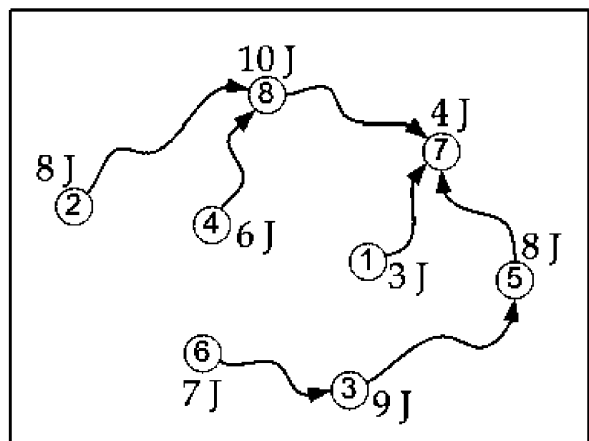

b Tree constructed by node 2

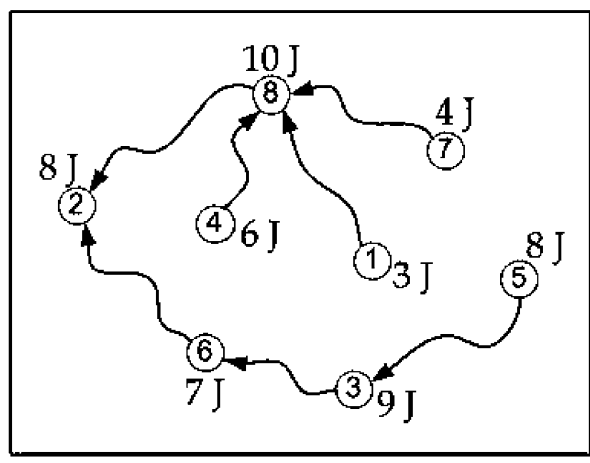

d $\quad$ Tree constructed by node 4

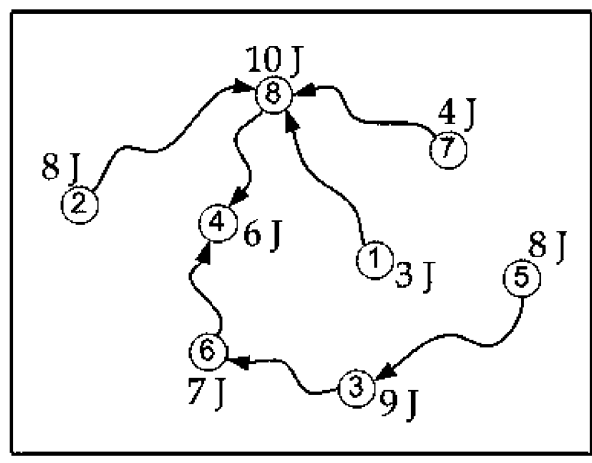

f $\quad$ Tree constructed by node 6

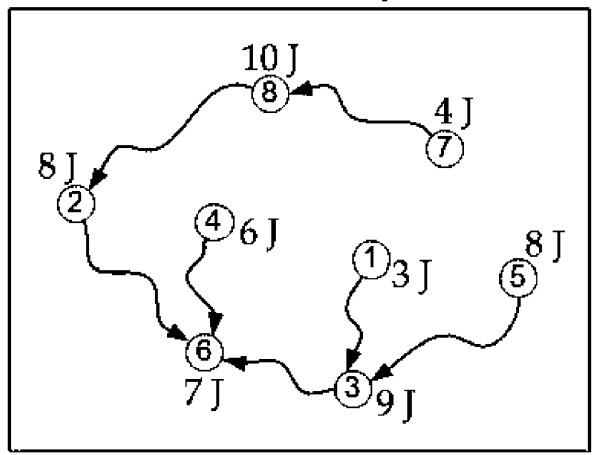

h $\quad$ Tree constructed by node 8

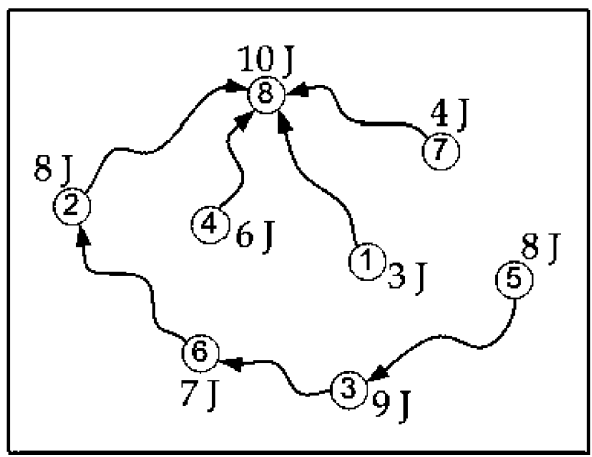

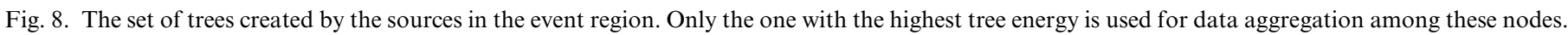
By using the SearchLPT function, all the sources will select the tree created and rooted at node 3 for aggregation.

To reasonably limit the simulation time, we altered the ns-2 energy model such that the sources carry an initial energy that is randomly chosen between 10 and
$15 \mathrm{~J}$. All other nodes (i.e., pure relaying nodes) are given with much higher initial energy so that the performance of the lifetime of the sources can be studied. 
Table 6

The SearchLPT Function

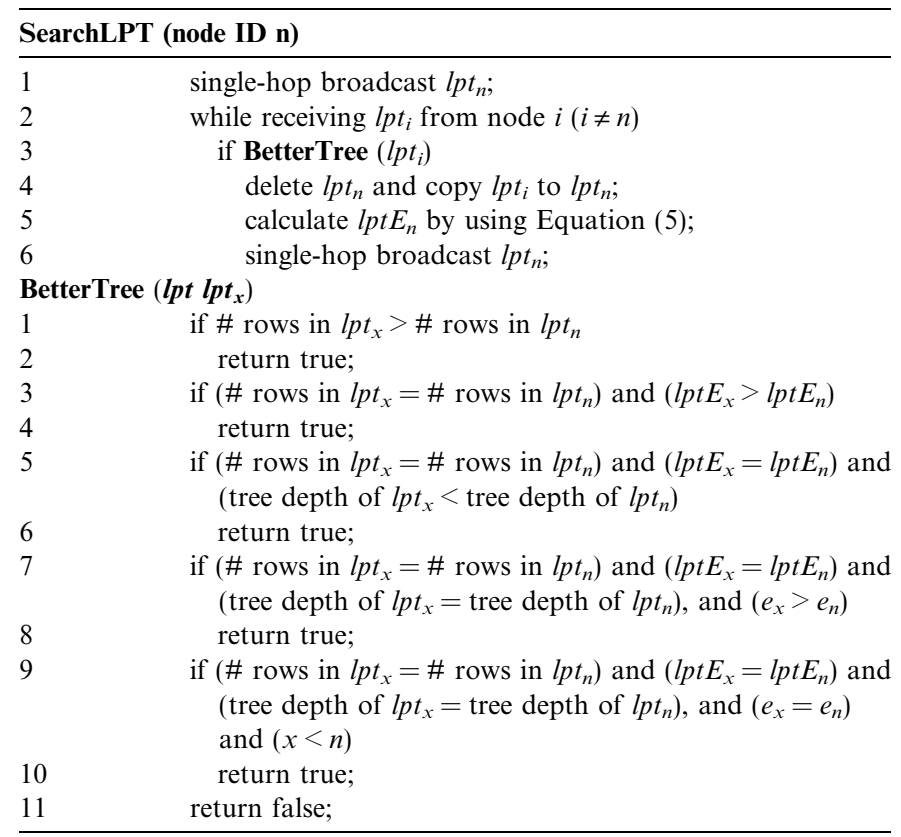

Idle time power, receive power, and transmit power dissipation are set at 35,395 , and $660 \mathrm{~mW}$, respectively. We assume that the data processing and aggregation cost are negligible.

\subsection{Tree energy: distributed LPT vs. centralized LPT}

Fig. 9 shows the percentage difference between the tree energy obtained by distributed and centralized LPT algorithms. The $x$-axis is the total number of sensor nodes $M$. Since we assume that the number of sources $N$ is always equal to $0.1 M$, an increase $M$ also increases $N$. Results show that the difference is less than $5 \%$. There is a slight increase in the percentage difference when $N$ increases. This is due to the fact that the distributed LPT algorithm sends broadcast messages during the tree construction phase. When packet loss occurs occasionally, the distributed tree may not be the same as the centralized one.

\subsection{Dissipated energy}

To study the impact of aggregation on energy reduction, we measure the average dissipated energy, which is defined as the average amount of energy consumed by a relaying sensor node throughout the entire simulation. The results are averaged over 20 different runs with a $95 \%$ confidence interval. Results in Fig. 10 show a considerable amount of energy reduction by using either LPT or E-Span when compared with Directed Diffusion. Such energy reduction is expected since both LPT and E-Span efficiently aggregate the data by combining several readings from various sources into a single summarized message.

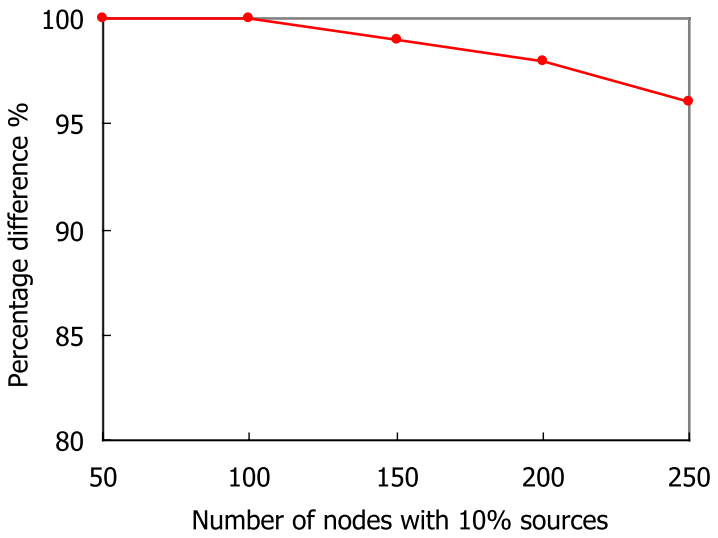

Fig. 9. Percentage difference on tree energy between distributed and centralized LPTs.

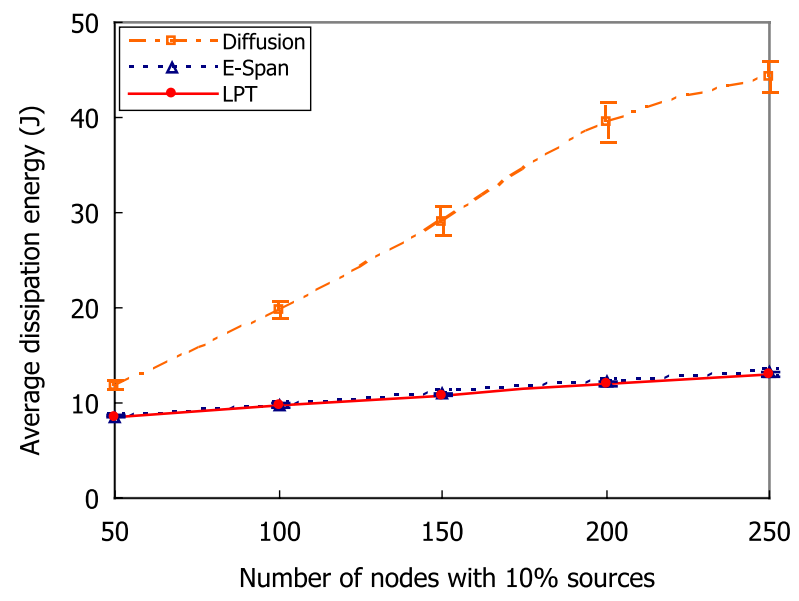

Fig. 10. Average dissipation energy as a function of network size.

\subsection{Node lifetime}

In order to study the impact of LPT and E-Span on lifetime improvement, we measure the node lifetime of each source as a function of network size for LPT, E-Span, and Directed Diffusion, respectively. The average node lifetime measures the time at which a source runs out of its available energy. Each node is assigned with an initial energy that is randomly chosen between 10 and $15 \mathrm{~J}$ so as to limit the total simulation time at a controllable range. Figs. 11-15 show the results when $M=50,100,150,200$, and 250 nodes, respectively. We make the following observations:

(1) Both LPT and E-Span considerably extend the node lifetime of each source, especially when the network is large.

(2) Both LPT and E-Span have similar performance when $M$ is small.

The impact of data aggregation is again validated in (1) above. By combining reports from various sources, both LPT and E-Span suppress a considerable amount of traffic in the network. Since less energy is being consumed, there 


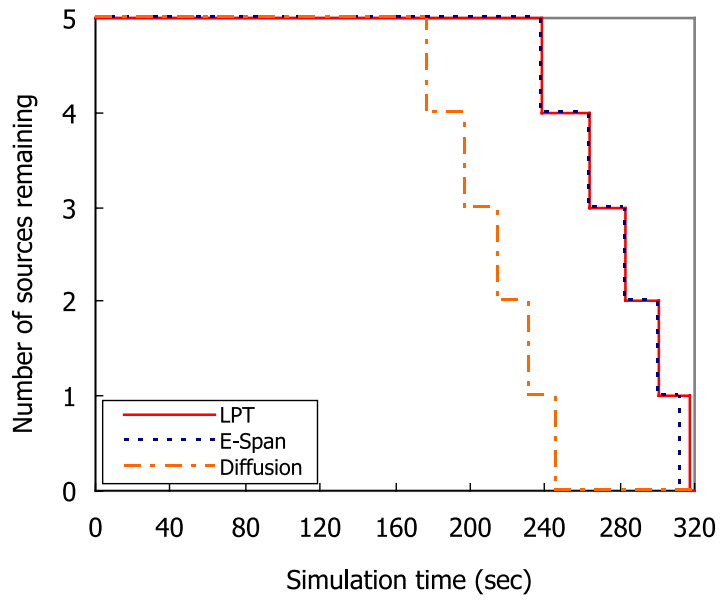

Fig. 11. Average node lifetime for each source with $M=50$ nodes.

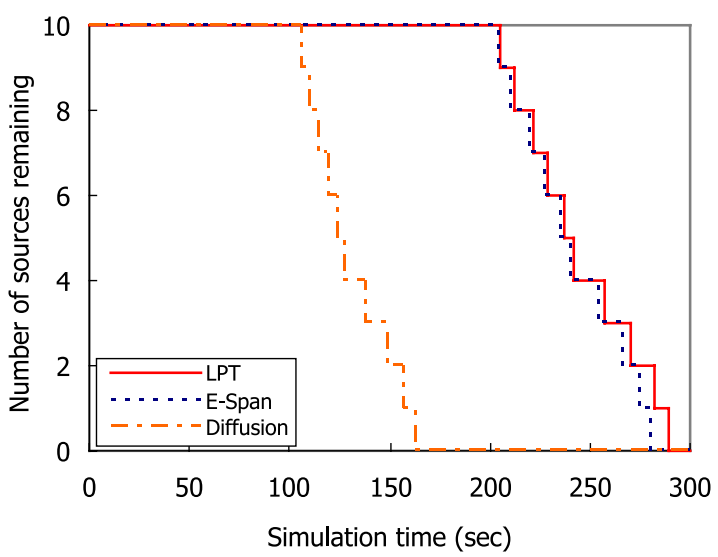

Fig. 12. Average node lifetime for each source with $M=100$ nodes.

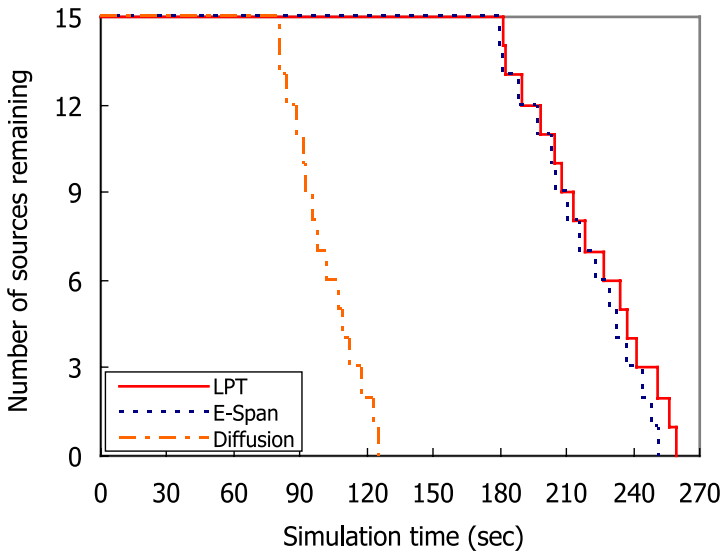

Fig. 13. Average node lifetime for each source with $M=150$ nodes.

is a noticeable lifetime improvement when data are collected via the trees. For (2), we argue that the chance of obtaining an identical tree by using LPT and E-Span, respectively, is relatively high when there are fewer sources. In fact, when all the nodes are within the radio range of each other, both LPT and E-Span will create an identical

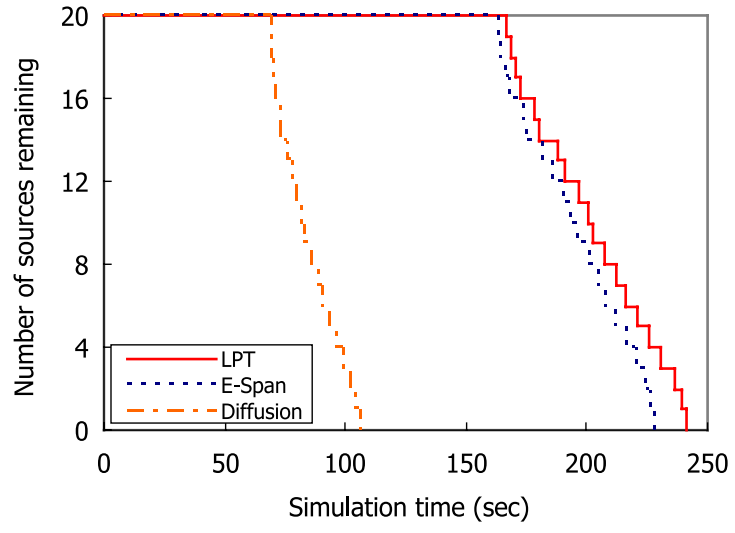

Fig. 14. Average node lifetime for each source with $M=200$ nodes.

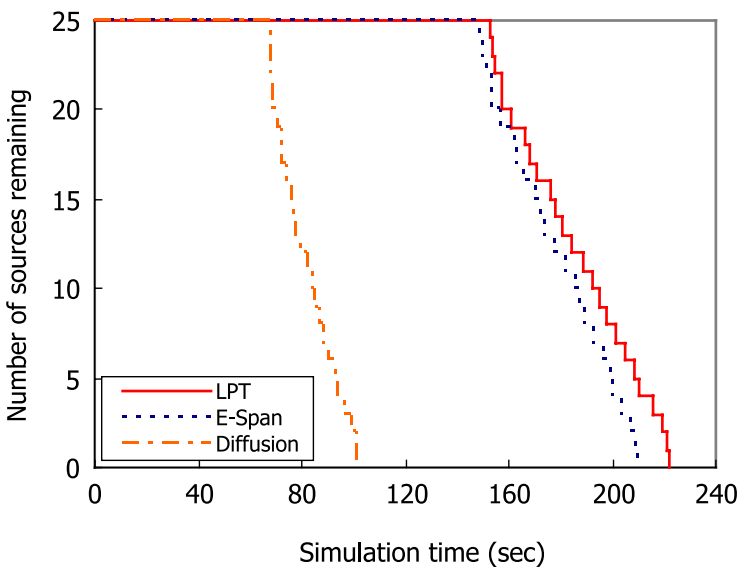

Fig. 15. Average node lifetime for each source with $M=250$ nodes.

tree with the highest-energy node selected as the root and all other nodes as leaf nodes. When this happens, the lifetime improvement will be similar. LPT has a better performance than E-Span when there are more sources within the event region.

Note that we choose a low initial residual energy and a small simulation time (less than $400 \mathrm{~s}$ ) in order to reduce the actual time to run all the simulations. Results reported

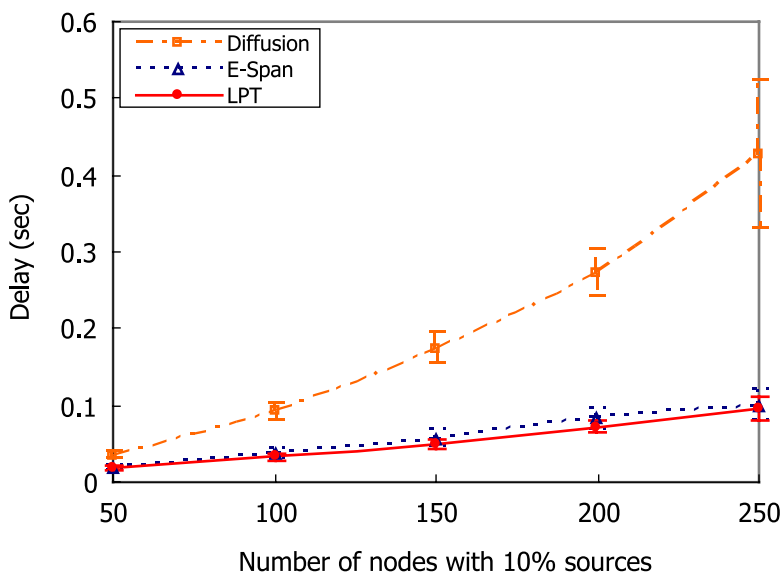

Fig. 16. Average data packet delay between a source and sink. 


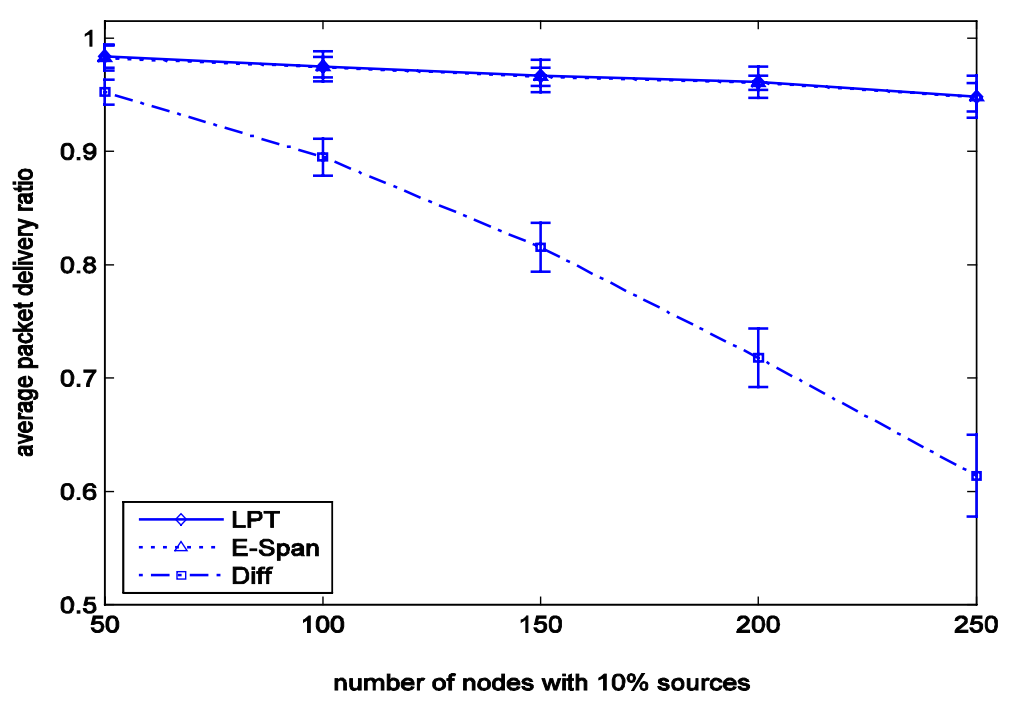

Fig. 17. Average packet delivery ratio between transmitting a data packet from each source and receiving it at each sink.

in this section can be extrapolated to the scenarios where the sensor nodes initial residual energy is much higher.

\subsection{Average data packet transfer delay}

The next experiment compares the average data packet delay between each source and sink for Directed Diffusion (Diff), LPT, and E-Span. Results in Fig. 16 show that both LPT and E-Span incur a lower delay than the Directed Diffusion. Since LPT and E-Span combine data from various sources, it is as if only a single source is generating packets. This is also true in a network with a large number of data sources.

\subsection{Average data packet delivery ratio}

The last experiment measures the average packet delivery ratio for Directed Diffusion (Diff), E-Span, and LPT. Fig. 17 shows that the Directed Diffusion experiences severe congestion when the number of sources $N$ increases. However, both LPT and E-Span are able to maintain their packet delivery ratios even when $N$ increases. The Directed Diffusion has its network overloaded with data traffic when more sources are sending packets. A considerable amount of data packets is therefore being dropped. LPT and E-Span, on the other hand, inject data to the sensor network as if there is only a single source. Thus, they are able to steadily maintain the packet delivery ratio even when the network is large.

\section{Conclusions}

In this paper, we proposed two overlay structures in which the sources within the event region form a tree to facilitate data aggregation. We first proposed E-Span, which is an energy-aware spanning tree algorithm. E-Span is a distributed protocol and facilitates the sources within an event region to perform data aggregation. In E-span, the source node which has the highest residual energy is chosen as the root. Each other node chooses its parent node among its neighbors based on the information of the residual energy and distance to the root. We also propose the Lifetime-Preserving Tree (LPT). Both the centralized and distributed LPT algorithms are described. Simulation results show that the trees created by both centralized and distributed LPT algorithms have similar tree energy. The average node lifetime for sensors using LPT are higher than that of the Directed Diffusion and E-Span. LPT also maintains a low average packet transfer delay and a high packet delivery ratio. Further work includes performance comparison between LPT with other data aggregation algorithms.

\section{Acknowledgment}

This work is supported by the Natural Sciences and Engineering Research Council of Canada under Grant No. 261604-03.

\section{References}

[1] C. Intanagonwiwat, R. Govindan, D. Estrin, Directed diffusion: a scalable and robust communication paradigm for sensor networks, in: Proc. of ACM MobiCom'00, Boston, MA, Aug. 2000, pp. 56-67.

[2] J.M. Kahn, R.H. Katz, K.S.J. Pister, Next century challenges: mobile networking for smart dust, in: Proc. of ACM MobiCom'99, Seattle, WA, Aug. 1999, pp. 271-278.

[3] G.J. Pottie, W.J. Kaiser, Wireless integrated network sensors, Commun. ACM 43 (5) (2000) 51-58.

[4] M. Ding, X. Cheng, G. Xue, Aggregation tree construction in sensor networks, in: Proc. of IEEE VTC'03, Orlando, FL, Oct. 2003, pp. 2168-2172.

[5] W. Zhang, G. Cao, DCTC: dynamic convoy tree-based collaboration for target tracking in sensor networks, IEEE Trans. Wireless Commun. 3 (5) (2004) 1689-1701.

[6] J. Carle, D. Simplot-Ryl, Energy-efficient area monitoring for sensor networks, IEEE Comput. Mag. 37 (2) (2004) 40-46. 
[7] S. Upadhyayula, V. Annamalai, S.K.S. Gupta, A low-latency and energy-efficient algorithm for convergecast, in: Proc. of IEEE GLOBECOM'03, San Francisco, CA, Dec. 2003, pp. 3525-3530.

[8] O. Younis, S. Fahmy, HEED: a hybrid, energy-efficient, distributed clustering approach for ad hoc sensor networks, IEEE Trans. Mobile Comput. 3 (4) (2004) 366-379.

[9] E.J. Duarte-Melo, M. Liu, A. Misra, A modeling framework for computing lifetime and information capacity in wireless sensor networks, in: Proc. of 2nd WiOpt: Modeling and Optimization in Mobile, Ad Hoc and Wireless Networks, Cambridge, UK, Mar. 2004.

[10] K. Dasgupta, K. Kalpakis, P. Namjoshi, An efficient clustering-based heuristic for data gathering and aggregation in sensor networks, in: Proc. of IEEE WCNC'03, New Orleans, LA, Mar. 2003, pp. 19481953.

[11] A. Sankar, Z. Liu, Maximum lifetime routing in wireless ad hoc networks, in: Proc. of IEEE Infocom'04, Hong Kong, Mar. 2004.

[12] E.J. Duarte-Melo, M. Liu, Analysis of energy consumption and lifetime of heterogeneous wireless sensor networks, in: Proc. of IEEE GLOBECOM'02, Taipei, Taiwan, Nov. 2002, pp. 21-25.

[13] V. Mhatre, C. Rosenberg, D. Kofman, R. Mazumdar, N. Shroff, A minimum cost heterogeneous sensor network with a lifetime constraint, IEEE Trans. Mobile Comput. 4 (1) (2005) 4-15.

[14] H. Zhang, J. Hou, On deriving the upper bound of $\alpha$-lifetime for large sensor networks, in: Proc. of ACM MobiHoc'04, Tokyo, Japan, May 2004, pp. 121-132.

[15] D.M. Blough, P. Santi, Investigating upper bounds on network lifetime extension for cell-based energy conservation techniques in stationary ad hoc networks, in: Proc. of ACM MobiCom'02, Atlanta, GA, Sept. 2002, pp. 183-192.

[16] T.H. Cormen, C.E. Leiserson, R.L. Rivest, C. Stein, Introduction to Algorithms, second ed., MIT Press, Cambridge, 2001.

[17] Y.K. Dalal, R.M. Metcalfe, Reverse-path forwarding of broadcast packets, Commun. ACM 21 (12) (1978) 1040-1048.

[18] VINT. (2001, Nov.) The network simulator ns-2. [Online]. Available: $<$ http://www.isi.edu/nsnam/ns/>.

[19] M. Lee, The construction of a lifetime-preserving tree for data aggregation in wireless sensor networks, M.A.Sc. Thesis, The University of British Columbia, Vancouver, Canada, Nov. 2004 (Available: $<$ http://www.ece.ubc.ca/ vincentw/T/MarcLee.pdf/>).
[20] M. Lee, V.W.S. Wong, An energy-efficient spanning tree algorithm for data aggregation in wireless sensor networks, in: Proc. of IEEE Pacific Rim Conference on Communications, Computers and Signal Processing (PacRim), Victoria, BC, Aug. 2005.

[21] M. Lee, V.W.S. Wong, LPT for data aggregation in wireless sensor networks, in: Proc. of IEEE Globecom'05, St. Louis, Missouri, Nov./ Dec. 2005.

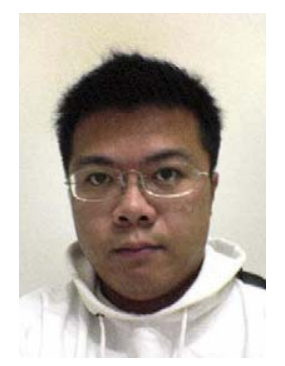

Weinan Marc Lee received the B.A.Sc. and M.A.Sc. degrees, both in electrical engineering, from the University of British Columbia, Vancouver, Canada, in 2002 and 2005, respectively. His research interests include wireless communications, wireless sensor networks, and passive RFID technologies.

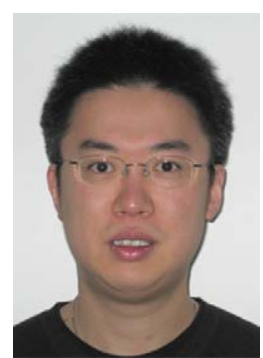

Vincent W.S. Wong received the B.Sc. degree from the University of Manitoba in 1994, the M.A.Sc. degree from the University of Waterloo in 1996, and the Ph.D. degree from the University of British Columbia (UBC) in 2000. He worked as a systems engineer at PMC-Sierra Inc from 2000 to 2001. He is currently an assistant professor in the Department of Electrical and Computer Engineering at UBC. His current research interests are in wireless sensor networks, wireless mesh networks, and heterogeneous wireless networks.

Dr. Wong received the Natural Sciences and Engineering Research Council postgraduate scholarship and the Fessenden Postgraduate Scholarship from Communications Research Centre, Industry Canada, during his graduate studies. He serves as TPC member in various conferences, including the IEEE Globecom'06 and ICC'06. 